\title{
LIN28B promotes the development of neuroendocrine prostate cancer
}

\author{
Jessica Lovnicki, ${ }^{1}$ Yu Gan, ${ }^{1,2}$ Tingting Feng, ${ }^{1,3}$ Yinan Li, ${ }^{1}$ Ning Xie, ${ }^{1}$ Chia-Hao Ho, ${ }^{1}$ Ahn R. Lee, ${ }^{1}$ Xufeng Chen, ${ }^{4}$ Lucia Nappi, ${ }^{1}$ Bo Han, ${ }^{3}$ \\ Ladan Fazli, ${ }^{1}$ Jiaoti Huang, ${ }^{4}$ Martin E. Cleave, ${ }^{1}$ and Xuesen Dong ${ }^{1}$
}

'Vancouver Prostate Centre, Department of Urologic Sciences, University of British Columbia, Vancouver, British Columbia, Canada. ${ }^{2}$ Department of Urology, Xiangya Hospital, Central South University, Changsha, China. ${ }^{3}$ Key Laboratory of Experimental Teratology, Ministry of Education, and Department of Pathology, School of Basic Medical Sciences, Shandong University, Jinan, China. ${ }^{4}$ Department of Pathology, Duke University School of Medicine, Durham, North Carolina, USA.

\begin{abstract}
Therapy-induced neuroendocrine prostate cancer (t-NEPC) is a highly aggressive subtype of prostate cancer with poor patient survival. Emerging evidence indicates that $t-N E P C$ can develop when prostate adenocarcinoma cells acquire cancer stem-like cell signaling in the presence of androgen receptor inhibition, followed by redifferentiation toward neuroendocrine lineage and subsequent t-NEPC progression. Whether the stem-like signaling is controlled by the core pluripotency stem cell genes (e.g., LIN28 and SOX2) remains unknown. Here, we report that the transcription of the LIN28B isoform and SOX2 were co-upregulated in t-NEPC patient tumors, patient-derived xenografts, transgenic mice, and cell models. Immunohistochemistry validated that LIN28B and SOX2 protein expression were elevated in t-NEPC patient biopsies. Using prostate adenocarcinoma and t-NEPC cell models, we demonstrated that LIN28B induced a stem-like gene network, neuroendocrine biomarkers, and neuroendocrine cell morphology. LIN28B depletion by CRISPR inhibited t-NEPC tumorigenesis and xenograft growth. These LIN28B functions were mediated mainly through the suppression of let-7 miRNA expression, resulting in de-repression of the transcription factor HMGA2 and HMGA2-mediated SOX2 expression. This study revealed a mechanism by which t-NEPC can develop through the LIN28B/let-7/SOX2 axis that regulates a cancer cell stem-like gene network, highlighting LIN28B as a potential therapeutic target in t-NEPC.
\end{abstract}

\section{Introduction}

New, potent androgen receptor (AR) pathway inhibitors (ARPIs) have improved overall survival in men with metastatic prostate cancer (PCa) (1, 2). However, more aggressive subtypes of castrate-resistant prostate cancer (CRPC) with low or no canonical AR signaling have become increasingly prevalent and account for approximately $20 \%$ of CRPC tumors $(3,4)$. Many of these tumors gain neuroendocrine (NE) and neural lineages after ARPIs and/or chemotherapies and progress to therapy-induced neuroendocrine prostate cancers (t-NEPCs) (5). Patients with t-NEPC have limited therapeutic options, and the median overall survival is less than 1 year (6). The molecular underpinnings of t-NEPC development are not fully understood, hindering the development of effective therapies to manage this disease.

The development of t-NEPC is regulated by PCa cell lineage plasticity, whereby prostate adenocarcinoma (AdPC) cells acquire a pluripotent stem-like phenotype followed by redifferentiation to NE lineage or undergo a transdifferentiation process to emerge as an $\mathrm{NE}$ lineage for t-NEPC tumorigenesis (5). These processes involve complex context-dependent mechanisms, including genomic alterations

Authorship note: JL and YG contributed equally to this work. Conflict of interest: The authors have declared that no conflict of interest exists. Copyright: () 2020, American Society for Clinical Investigation. Submitted: December 5, 2019; Accepted: June 25, 2020; Published: August 31, 2020. Reference information: / Clin Invest. 2020;130(10):5338-5348. https://doi.org/10.1172/JCl135373.
(7-10), epigenetic reprogramming (11-13), alternative RNA splicing $(12,14)$, and aberrant activation of transcriptional factors $(7,13-15)$. The diversity of genomic backgrounds and variability of molecular pathways help explain the heterogeneity of t-NEPC (16) and multiple driver genes discovered to date (7-15). These findings suggest that AdPC cells can exploit multiple possible signaling pathways to drive t-NEPC progression. Depending on their genomic backgrounds and tumor microenvironments, patients' tumors may use a variety of mechanisms that confer survival and growth advantages to bypass pressures of ARPIs and support treatment resistance. These findings underscore that using therapies in combination will be most beneficial for patients with t-NEPC. Since the pluripotent stem-like phenotype gives PCa cells the flexibility to adapt various survival pathways for the development of therapy resistance, understanding the regulatory mechanisms by which stem-like gene networks promote $\mathrm{t}$-NEPC is imperative for designing therapies and optimizing the control of intertumor and intratumor heterogeneity.

The most well-defined core "stemness" genes in human cells are the SOX2, LIN28, POU5F1, and NANOG genes, which are powerful enough to reprogram terminally differentiated human fibroblasts into induced pluripotent stem cells (17). They have been shown to be biomarkers of cancer stem cells (CSCs) and important for developing therapy resistance in tumors (18-22). These findings imply that these stemness genes may play a key role in t-NEPC progression. It has been reported that when AdPC cells with genomic disruptions of the PTEN, TP53, and RB1 genes 
were treated with ARPIs, they developed stem-like, neural, and epithelial-mesenchymal transition (EMT) phenotypes and progressed to t-NEPC in part mediated by SOX2 (7-10). Mutual regulation between SOX2 and LIN28 was reported in a cell context-dependent manner $(23,24)$. In squamous carcinoma cells, LIN28B enhances SOX2 expression through increased transcriptional factors of HMGA2 and ARID3B that are de-repressed by let-7 miRNA (23). Conversely, in neural precursor cells, SOX2 binds to the LIN28 promoter and induces LIN28 transcription (24). Together, these findings suggest that LIN28 and SOX2 may regulate a stem-like gene network to confer PCa cell lineage plasticity for t-NEPC development.

Human cells have 2 LIN28 homologs (LIN28A and LIN28B) that are usually not coexpressed in the same cell lineage (25). They are both RNA binding proteins that share a high degree of homology in their functional domains. Their best characterized functions are to prevent Dicer and Drosha from processing pri- and pre-let-7 miRNAs to generate mature let-7 miRNA (26). LIN28 was also reported to bind mRNAs and further recruit RNA helicase A to facilitate translation (27-29). In this study, we report that LIN28B and SOX2 were co-upregulated in a subgroup of t-NEPC patient tumors, t-NEPC xenografts, genetically engineered mouse models (GEMMs), and t-NEPC cell models. LIN28B regulated a stem-like gene network to promote t-NEPC development, whose function can be mediated by inhibition of let-7 miRNA and de-repression of HMGA2 followed by HMGA2-mediated SOX2 transcription. These findings revealed a molecular mechanism by which t-NEPC development can be developed through the LIN28B/let-7/SOX2 axis and identified LIN28B as a potential therapeutic target for t-NEPC.

\section{Results}

LIN28B expression is upregulated in $t$-NEPC. The role of SOX2 in mediating a neural stem cell phenotype to promote t-NEPC development highlights the clinical significance of the stem-like gene network $(7,8)$. Because SOX2 is 1 of the 4 core "stemness" genes that can reprogram fully differentiated fibroblast cells into induced pluripotent stem cells, we analyzed the RNA-Seq data of these genes in CRPC patient samples in association with t-NEPC from the Beltran 2016 cohort (30). While NANOG, POU5F1, and $L I N 28 A$ were expressed at low levels in these tumors, $L I N 28 B$ was distinctly expressed in the t-NEPC group (Figure 1A and Supplemental Figure 1A; supplemental material available online with this article; https://doi.org/10.1172/JCI135373DS1). Seven t-NEPC samples expressed low levels of LIN28B (fragments per kilobase of exon model per million reads mapped read <0.3), similar to that of the AdPC samples, and the remaining $8 \mathrm{t}$-NEPC samples expressed an approximately 98.6-fold increase in LIN28B levels. This LIN28B expression pattern overlapped with that of SOX2, signifying a potential positive correlation between LIN28B and SOX2, where they may act as key regulators in a pluripotency network to promote a neural lineage fate.

We also found a significant increase in both LIN28B and SOX2 expression in the DuNE, but not the LnNE, t-NEPC cell model (Figure 1A). DuNE and LnNE cells are both transformed t-NEPC cells by the neural RNA splicing factor SRRM4 from DU145 and LnCaP cell lines, respectively $(31,32)$. Although both t-NEPC models have similar NE gene signatures to t-NEPC patient sam- ples $(31,32)$, the differential expression of LIN28B and SOX2 in these 2 models mimics the LIN28B ${ }^{\text {hi }}$ and LIN28B $B^{\text {lo }} \mathrm{t}-\mathrm{NEPC}$ patient tumors. These findings suggest that the DuNE and LnNE cell models are developed through different mechanisms of either a pluripotency stem-like signaling or a transdifferentiation process (32). Together, these results indicated that there exist different subgroups of t-NEPC, where the division of the 2 subgroups can be based on the distinct expression of LIN28B and SOX 2 .

Induction of $L I N 28 B$ expression was also observed in other t-NEPC models. There were positive associations of LIN28B with t-NEPC in the Living Tumor Laboratory patient-derived xenograft (LTL PDX) model (ref. 33 and Figure 1B). Particularly, the precastrated LTL331 xenografts showed a classic AdPC phenotype but were transformed into castration-resistant t-NEPC LTL331R tumors, during which LIN28B was markedLY upregulated and positively associated with SOX2 and SYP and negatively with AR expression (Figure 1C). Consistently, the negative association of LIN28B expression with AR was observed in the Beltran 2016 and SU2C 2015 cohorts (Supplemental Figure 1, B and C). RNA-Seq results from the GEMMs under ARPI treatment showed that both WT and PTEN knockout (single knockout, SKO) mice (8) expressed extremely low LIN28B, which was in contrast to the tumors from the PTEN/TP53 (double knockout, DKO) and PTEN/TP53/RB1 (triple knockout, TKO) mice that expressed high LIN28B (Figure 1D). After treatment of PTEN/TP53-KO mice with abiraterone (9), LIN28B expression was significantly increased in the overt $\mathrm{NE}$, but not in the focal $\mathrm{NE}$ mouse tumor samples (Figure 1E). Collectively, these results indicated that LIN28B upregulation in t-NEPC cells was associated with AR inhibition in a genomic context-dependent manner.

To conduct functional analyses of LIN28B in PCa cell models, we measured LIN28B expression in various cell lines and found that LIN28B was highly expressed in the DuNE, NCI-H660, LASCPC1 NEPC lines, and NCI-H69 and NCI-H82 small cell lung cancer lines by quantitative PCR (qPCR) and immunoblotting (Figure 1, F and G). These results were consistent with the data reported by the Cancer Cell Line Encyclopedia (ref. 34 and Supplemental Figure 1D). When a luciferase reporter vector containing a LIN28B gene promoter (35) was introduced to these cell lines, we found that it was active in the DuNE and NCI-H69, but not the DU145 and LnCaP and LnNE cell lines (Figure 1H), suggesting that the induction of LIN28B was regulated at the transcription level. Collectively, these results linked the upregulation of LIN28B expression with t-NEPC.

LIN28B and SOX2 expression are co-upregulated in a subgroup of $t$-NEPC. To confirm LIN28B and SOX2 protein expression in t-NEPC using tumor samples from an independent patient cohort, we applied IHC on tissue microarrays (TMAs) from the Vancouver Prostate Centre (VPC) tissue bank containing 16 primary AdPC, 54 CRPC-AdPC, and 30 CRPC-NEPC tissue cores that were previously characterized by histology, NE, and AdPC marker status $(12,36,37)$. The specificity of the LIN28B antibody for IHC was confirmed by using tissue slides with known LIN28B mRNA and protein status (Figure 1I). Cell lysis fragmentation combined with immunoblotting assays indicated that LIN28B was localized in the cytoplasm of PCa cells (Supplemental Figure 2A), supporting the scoring of cytoplasmic staining by pathologists. IHC optimization of the SOX2 antibody was reported previously (32). 

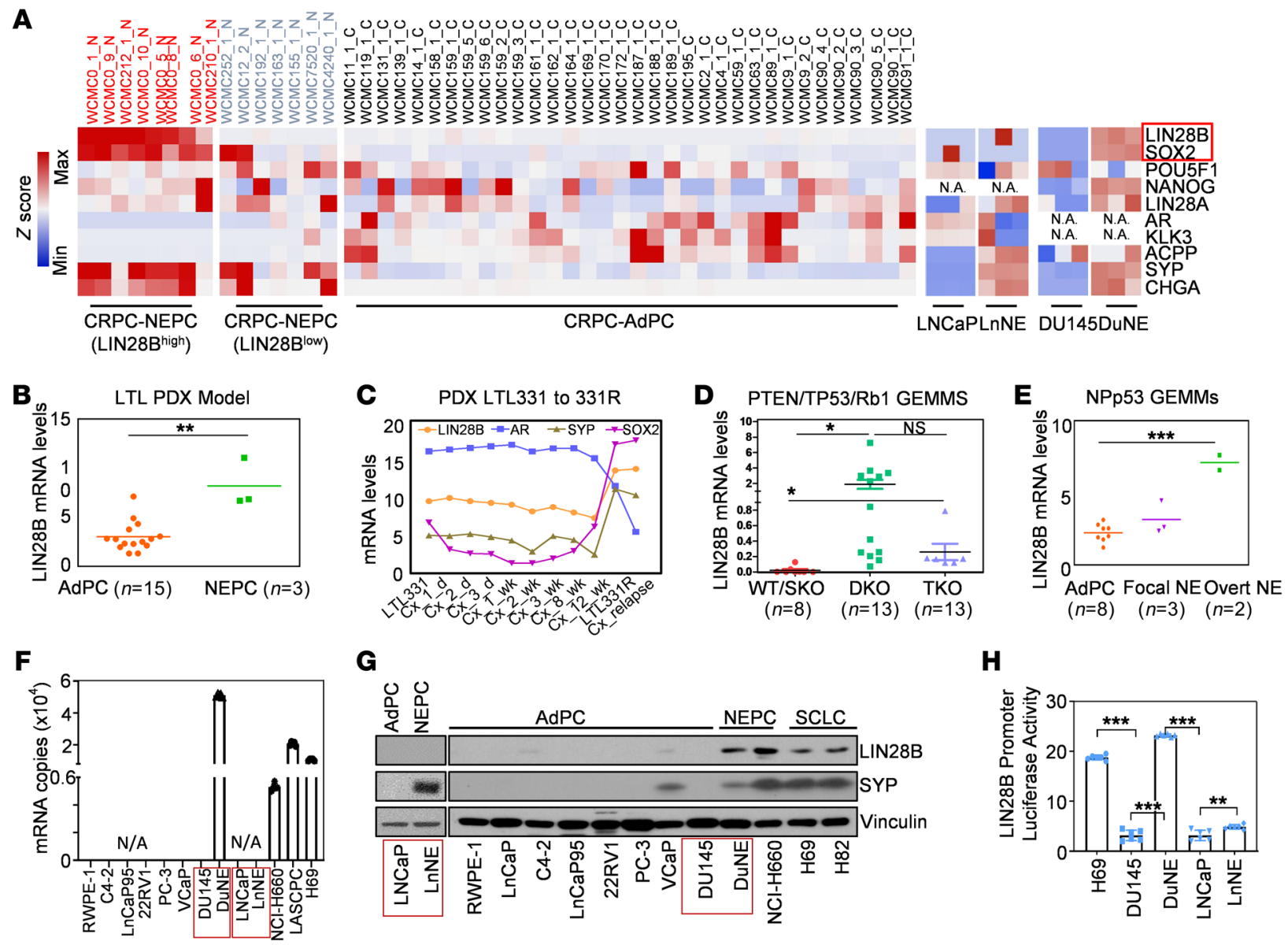

G

\section{H}
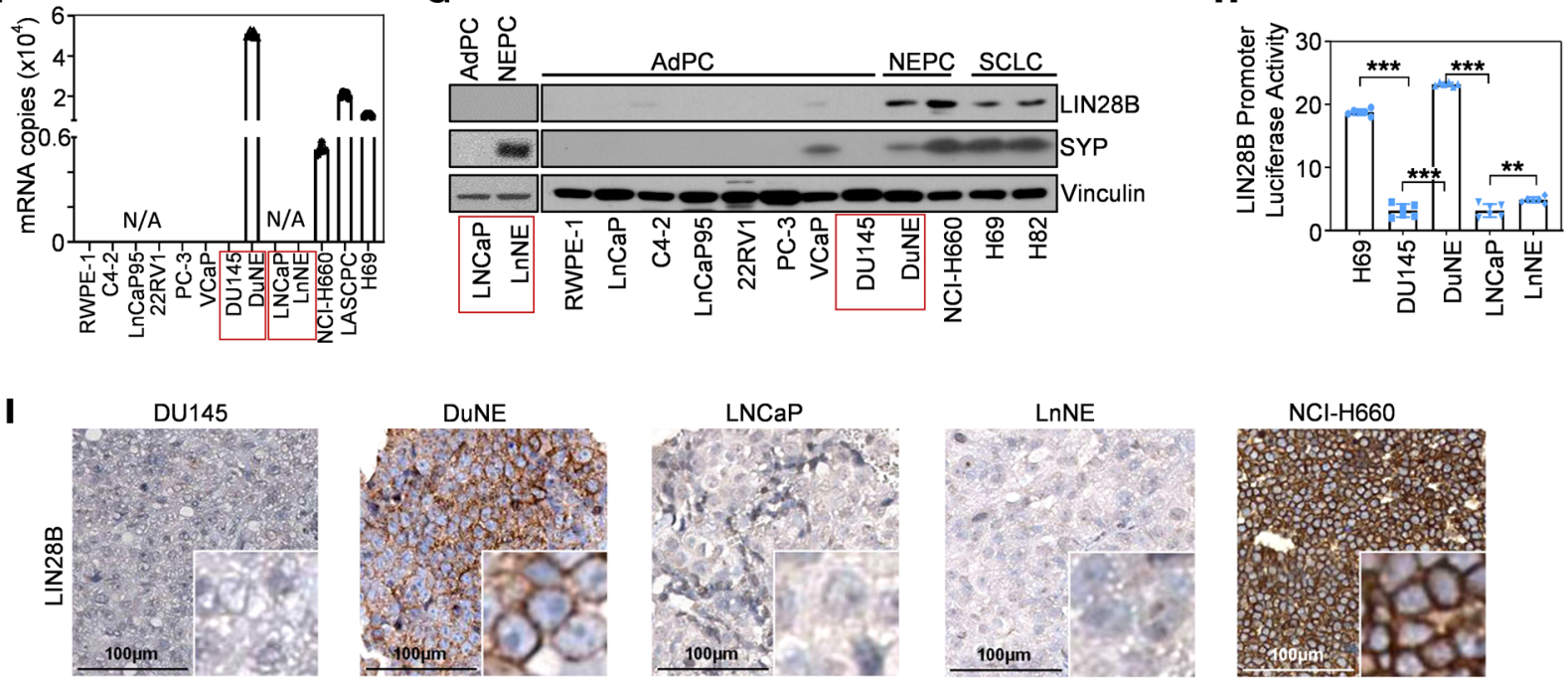

Figure 1. LIN28B expression is upregulated in t-NEPC. (A) RNA-Seq results of the 4 core stemness genes, NE, and epithelium biomarkers from the Beltran 2016 cohort $(n=49)(30)$ and the indicated cell models (31, 32) were plotted. (B) The mRNA levels of LIN28B in 15 AdPC and 3 NEPC PDX models (64) were plotted. (C) The mRNA levels of LIN28B, SOX2, AR, and SYP during AdPC (LTL331) progression to t-NEPC (LTL331R) by castration surgery to the host mice (33) were plotted. (D) LIN28B RNA-Seq results from the GEMMs (8) were plotted. SKO, single PTEN knockout; DKO, PTEN plus TP53 double knockout; TKO, PTEN, TP53, plus Rb1 triple knockout. (E) LIN28B expression in different phenotypic subcategories of the NPp53 GEMMs (9) is shown. (F and G) LIN28B levels in AdPC, NEPC, and SCLC cell lines was measured by real-time qPCR and immunoblotting. (H) Indicated cell lines were transfected with a luciferase reporter vector to measure LIN28B promoter activity. (I) IHC was performed on xenograft tissue slides using the LIN28B antibody. Scale bar: $100 \mu \mathrm{m}$. The real-time PCR and immunoblotting experiments were performed in 3 independent technical replicates. Results are presented as mean \pm SD and statistical analyses were performed by 1-way ANOVA or unpaired Student's $t$ test with ${ }^{*} P<0.05,{ }^{* *} P<0.01$, and ${ }^{* * *} P<0.001$. The red boxes highlight 2 extremely opposite phenotypes of cells derived from the same parental cell line.

IHC scores of both LIN28B and SOX2 were significantly higher in a subgroup of t-NEPC $(P<0.001)$ (Figure 2, A and B). We found that LIN28B and SOX2 were highly expressed in 14 out of 30 and 15 out of $30 \mathrm{t}$-NEPC tissue cores, respectively. When the t-NEPC cores were subdivided into t-NEPC (LIN28B ${ }^{\text {lo }}$ (IHC score $<0.4$ ) and t-NEPC(LIN28B ${ }^{\text {hi }}$ ) (IHC score $\geq 0.4$ ) groups, we found that SOX2 expression was highly correlated with LIN28B, with a Pearson $r$ correlation coefficient of $0.6(P<0.001)$ (Figure 2B). This finding was consistent with the RNA-Seq results from both the Beltran 2016 and SU2C 2015 cohorts (Supplemental Figure 2, B and C). To further elucidate the causal relationship between LIN28B and SOX2 in t-NEPC, we designed 3 shRNA sequences against LIN28B that efficiently knocked down LIN28B mRNA levels more than $50 \%$ and significantly reduced SOX2 mRNA and protein levels in DuNE cells (Figure 2C). However, RNA silencing of SOX2 did not reduce LIN28B expression. Furthermore, SOX2 overexpression in DU145 cells did not increase LIN28B expression, indicating that LIN28B was an upstream regulator of SOX2 
A
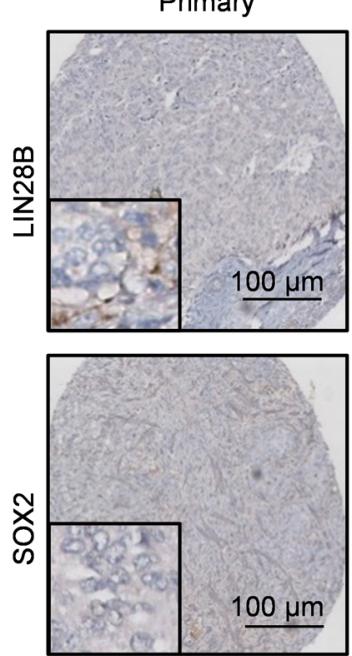

CRPC-AdPC
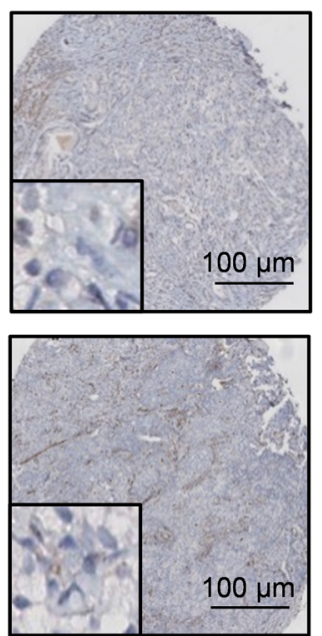

NEPC LIN28B Low
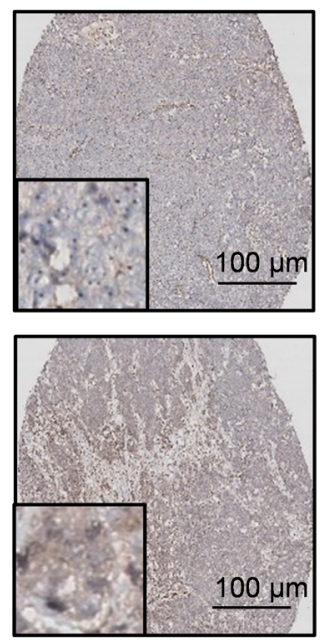

NEPC LIN28B high
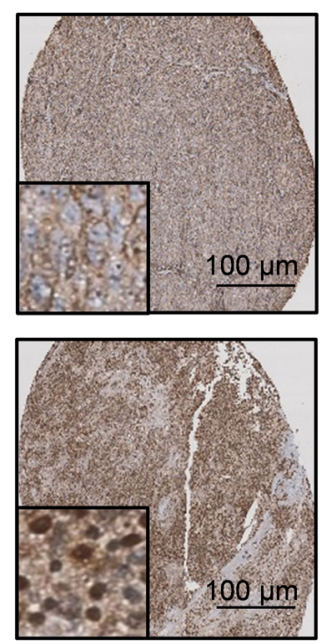

B
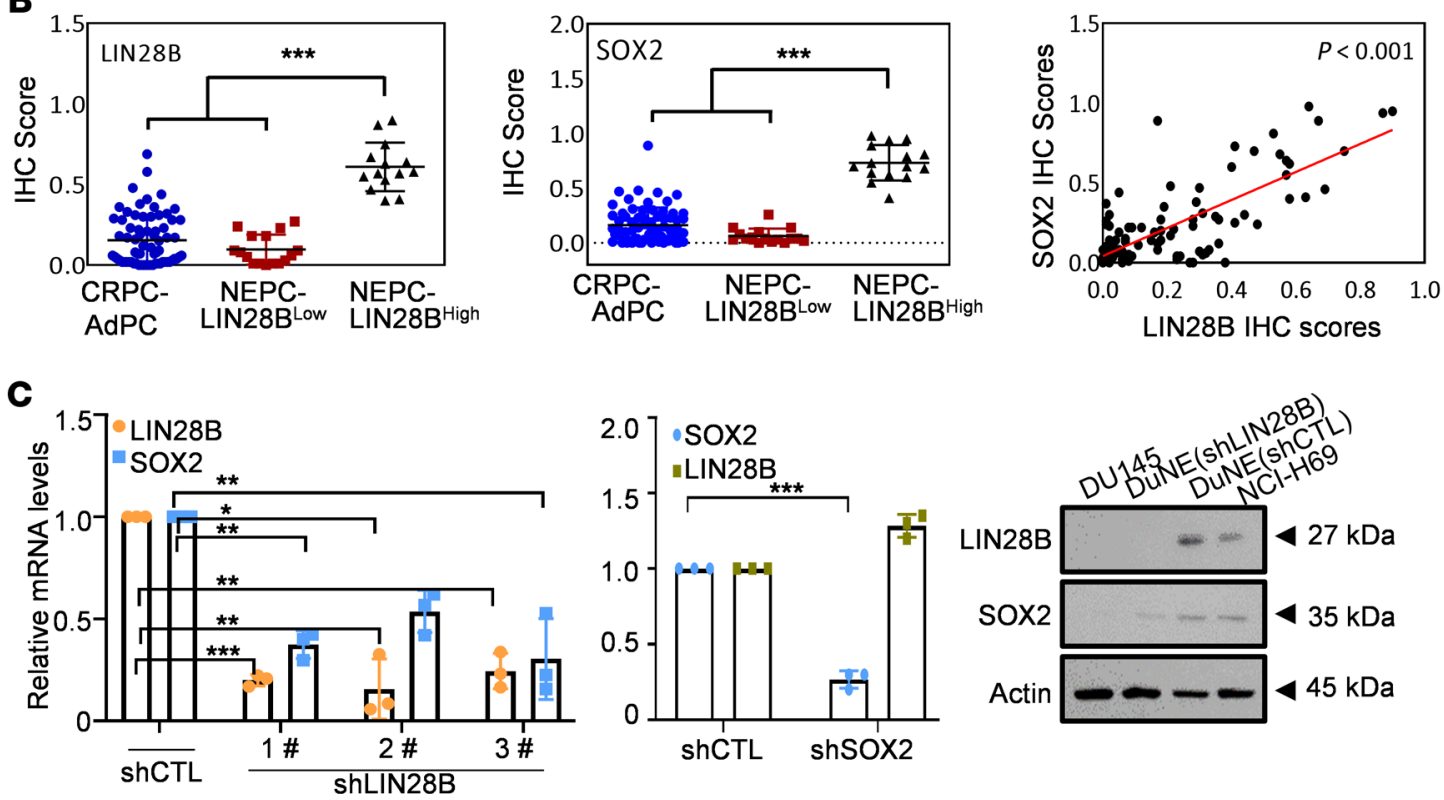

Figure 2. LIN28B and SOX2 expression are co-upregulated in a subgroup of t-NEPC. (A) IHC was performed on a CRPC patient TMA with LIN28B and SOX2 antibodies. Tissue cores were grouped into primary tumors $(n=16)$, CRPC-AdPC $(n=54)$, t-NEPC(LIN28B $\left.{ }^{10}\right)(n=16)$, and t-NEPC (LIN28B ${ }^{\text {hi) }}$ ( $\left.n=14\right)$, with 1 representative image from matched tissue cores from each group shown. Scale bar: $100 \mu \mathrm{m}$. (B) IHC scores of LIN28B and SOX2 were plotted. Pearson $r$ correlation coefficient between LIN28B and SOX2 expressions is 0.60. (C) DuNE cells were transfected with control or 3 different shRNAs against LIN28B or the previously reported siRNA against SOX2 (32). Real-time PCR and immunoblotting assays measured LIN28B and SOX2 expression. Three independent technical replicates were performed for each real-time PCR and immunoblotting experiment. Only 1 set of representative immunoblots is shown. All results are presented as mean \pm SD. Statistical analyses were performed by 1-way ANOVA or unpaired Student's $t$ test with ${ }^{*} P<0.05,{ }^{* *} P<0.01$, and ${ }^{* * *} P<0.001$, respectively.

(Supplemental Figure 2D). Together, these results demonstrated that LIN28B and SOX2 were co-upregulated in a subgroup of $\mathrm{t}$-NEPC, suggesting that these 2 genes may induce a pluripotency stem-like signaling during t-NEPC development.

LIN28B regulates a stem-like pluripotency gene network in $t-N E P C$ cells. To study the function of LIN28B in t-NEPC cells, we constructed cell models by applying CRISPR/Cas9 to knock out the LIN28B gene in DuNE cells. Since DuNE cells have multiple copies of the LIN28B gene, 3 monoclones were selected that had a significant but not complete deletion of the LIN28B gene, as confirmed by immunoblotting and Sanger sequencing (Supplemental Figure 3, A and B). Clone 3, designated as DuNE(gLIN28B), was used in the Ion AmpliSeq Transcriptome analyses where we profiled the differential transcriptome between DuNE(gCTL) and DuNE(gLIN28B) containing 3302 genes. When analyzed by DAVID 6.8 (https://david.ncifcrf.gov) for unbiased functional annotation, we found that these genes were associated with cellular processes, such as nucleic acid binding, transcription, and ubiquitin-dependent protein catabolic process, and development processes, such as embryonic morphogenesis, neural differentiation, regulation of cell proliferation, and axon guidance. Further gene set enrichment analysis (GSEA) showed that LIN28B-regulated genes were associated with cell lineage plasticity and embryogenesis (Fig- 

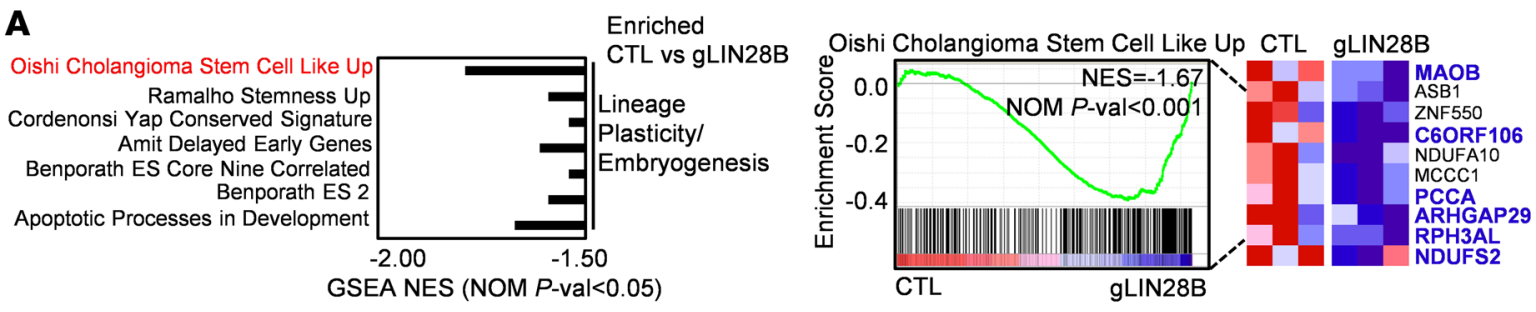

B
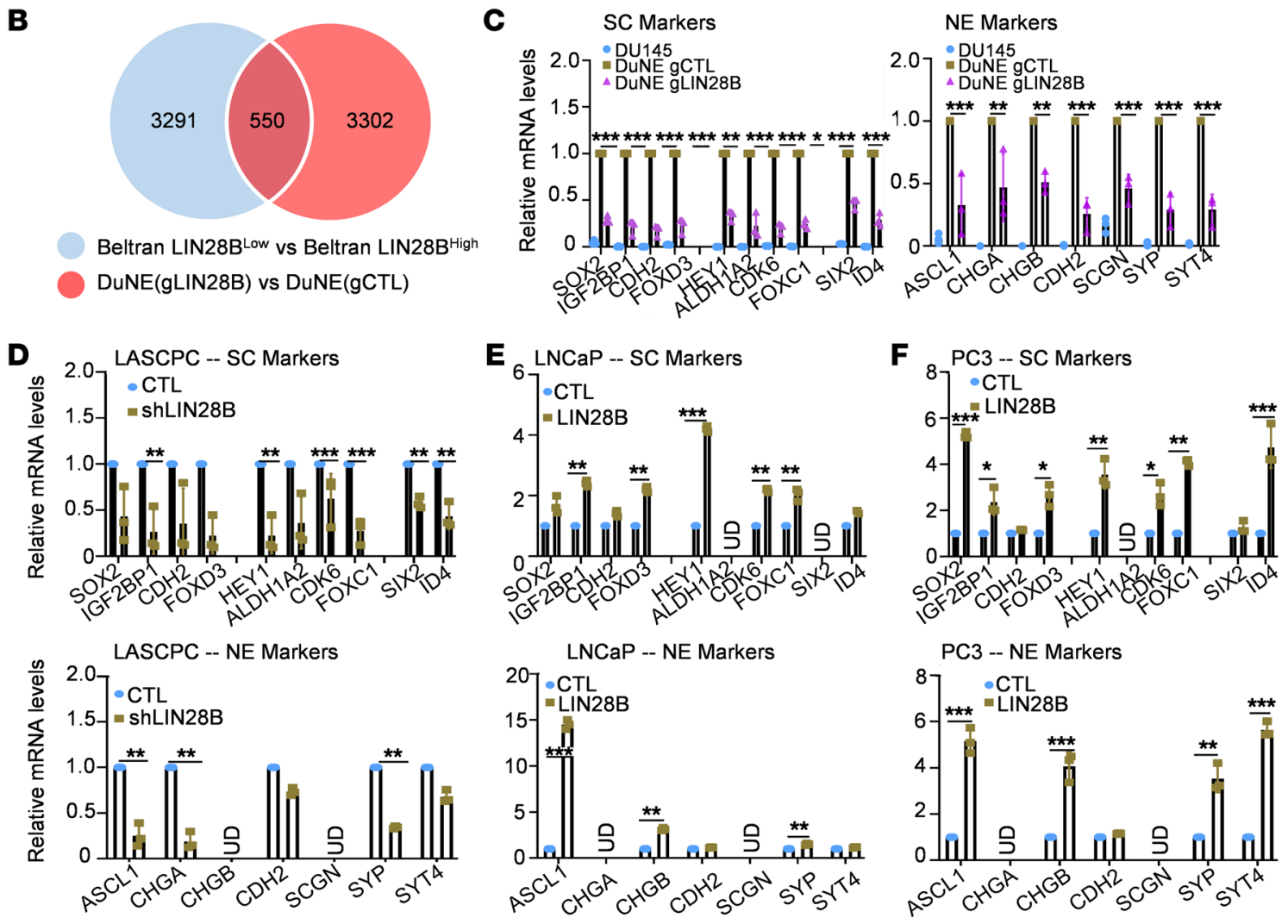

Figure 3. LIN28B regulates a stem-like pluripotency gene network in t-NEPC cells. (A) Differentially expressed genes between DuNE(gCTL) and DuNE(gLIN28B) cells ( $P$ value cut off was 0.05 ) were analyzed by GSEA. Cenes in blue were also upregulated in the LIN28B group from the Beltran patient cohort (30). (B) Differentially expressed genes between DuNE(gCTL) and DuNE(gLIN28B) cells overlapped with the differentially expressed genes between LIN28B ${ }^{\text {hi }}$ and LIN28B ${ }^{\circ}$ patient samples from the Beltran cohort. A total of 550 genes were identified to be in common. (C-F) Real-time PCR measured the mRNA levels of stem cell (SC) and neuroendocrine (NE) markers in DU145, DuNE(gCTL), and DuNE(gLIN28B) cells (C), LASPC1 cells with/without shLIN28B (D), LNCaP (E), and PC3 (F) cells with/without LIN28B overexpression. Three independent technical replicates were performed for each real-time PCR experiment. All results are presented as mean \pm SD. Statistical analyses were performed by 1-way ANOVA or unpaired Student's $t$ test with ${ }^{*} P<0.05,{ }^{* *} P<0.01$, and ${ }^{* * *} P<0.001$.

ure 3A). For example, LIN28B depletion was negatively associated with Oishi Cholangioma Stem Cell Like Up, Ramalho Stemness Up, and Benporath ES 2 gene signatures (Supplemental Figure 4). To stratify the t-NEPC-relevant LIN28B-regulated genes, we further overlapped the 3302 genes with the differential transcriptome between t-NEPC (LIN28B ${ }^{\text {lo }}$ ) and t-NEPC(LIN28B $\left.{ }^{\text {hi }}\right)$ tumors from the Beltran 2016 patient cohort and found 550 gene alterations were in common (Figure 3B). The top 10 ranked genes suppressed in the DuNE(gLIN28B) cells were listed with 6 genes from the Beltran t-NEPC $\left(\right.$ LIN28B ${ }^{\text {lo }}$ ) subgroup (Figure 3, A and B). To validate these gene annotation analyses, we performed qPCR to show that DuNE cells expressed high levels of stem-like and NE markers that could be strongly inhibited by LIN28B depletion (Figure 3C); these results were replicable in LIN28B-positive LASCPC1 cells (Figure 3D). Additionally, LIN28B overexpression in LIN28B-negative LNCaP, PC3, and DU145 cells, as well as LNCaP-derived enzalutamide-resistant MR42D and MR42F cells, significantly upregulated stem-like and NE markers (Figure 3, E and F, and Supplemental Figure 5, A-C). Collectively, these results confirmed that LIN28B regulated a stemlike gene signaling that could reprogram the AdPC phenotype toward stem-like and NE phenotypes.

Based on the gene signature regulated by LIN28B, we further validated whether LIN28B confers a stem-like phenotype in PCa cells. Flow cytometry assays using antibodies against 2 stem cell biomarkers, CD44 and CD133, showed that LIN28B depletion in DuNE cells significantly reduced $\mathrm{CD} 44^{+}$and $\mathrm{CD} 133^{+}$cell populations in comparison with the DuNE control cells (Figure 4A). While the DuNE cells grew as 3D spheroids on Matrigel, LIN28B depletion resulted in monolayer adherent epithelial 2D morphology (Figure 4B). LIN28B overexpression in DU145 cells, PC3, LNCaP, MR42F, 
A

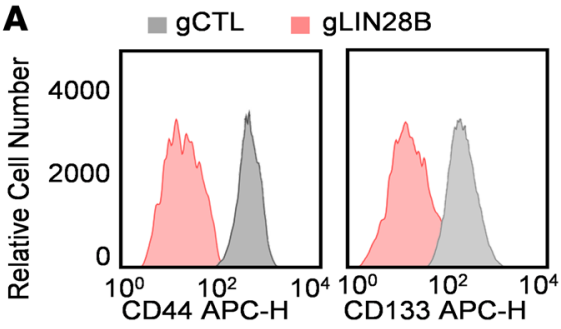

D

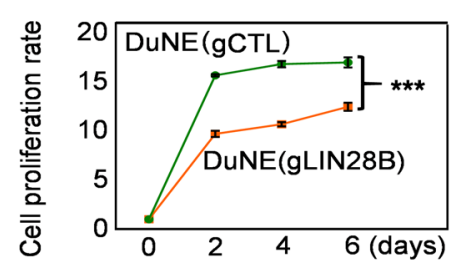

B

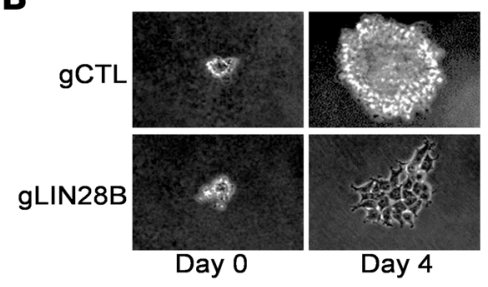

E

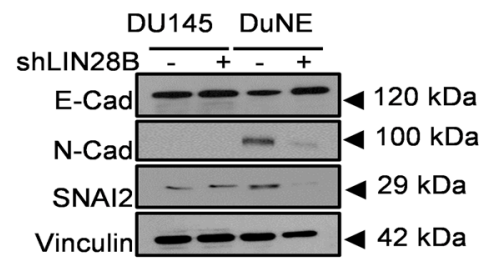

C

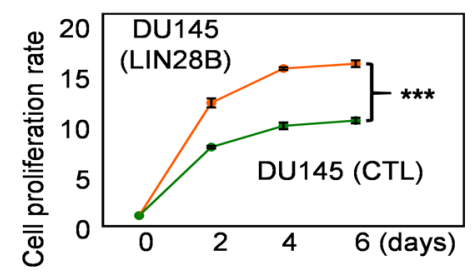

F

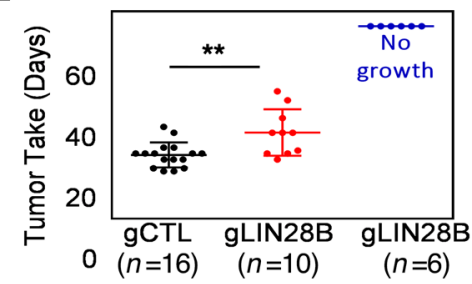

H

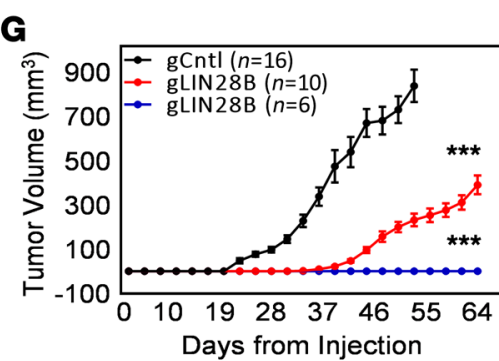

I

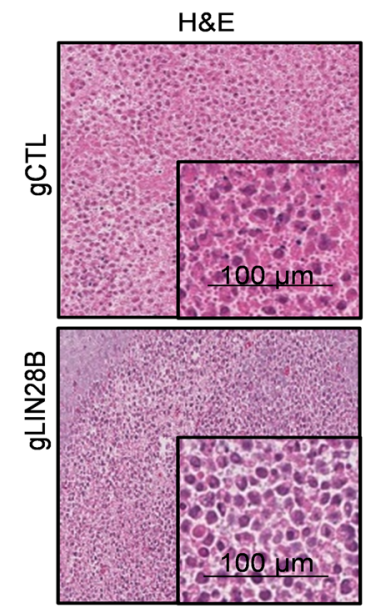

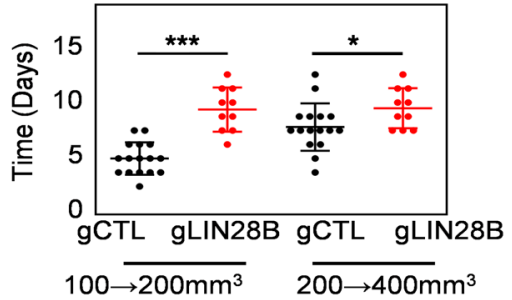

LIN28B
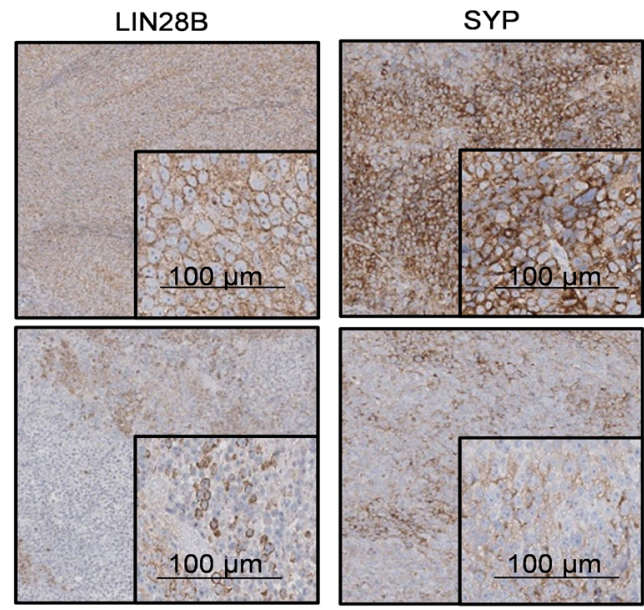

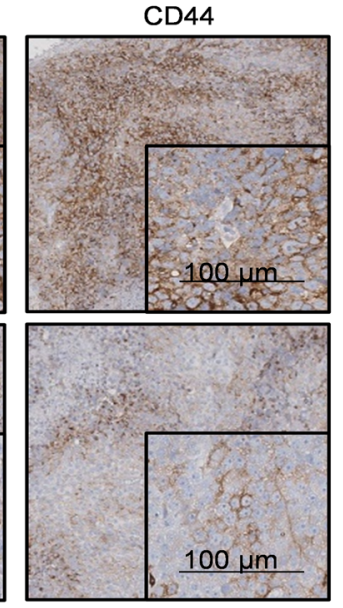

Figure 4. LIN28B regulates a stem-like phenotype in t-NEPC cells. (A) FACS analyses measured CD44+ and CD133+ cell populations regulated by LIN28B knockout. (B) DuNE(gCTL) and DuNE(gLIN28B) cells were used to perform colony formation assays. (C and D) MTS assays measured cell proliferation of DU145 cells with/without IN28B overexpression and DuNE cells with/without LIN28B knockout. (E) E-cadherin, N-cadherin, and SNAI2 protein levels were measured by immunoblotting. (F) Tumor take rates and (G) tumor volume and tumor doubling time of DuNE(gCTL) and DuNE(gLIN28B) xenografts were plotted. (H and I) LIN28B, SOX2, SYP, CD44, and N-Cad expressions from the xenografts were measured by immunoblotting and IHC. All FACS, qPCR, and immunoblotting assays were repeated in triplicate. The Student's $t$ test was used to compare results between 2 groups with ${ }^{*} P<0.05,{ }^{* *} P<0.01$, and ${ }^{* * *} P<0.001$, respectively.

and MR42D cells increased, whereas LIN28B depletion in DuNE cells reduced cell proliferation (Figure 4, C and D, and Supplemental Figure 6). We also found that the DuNE cells possessed an EMT phenotype, consistent with the high metastatic property of t-NEPC cells. However, LIN28B depletion reduced EMT markers N-cadherin and SNAI2 but increased E-cadherin expression (Figure 4E) and decreased cell migration (Supplemental Figure 7). Together, these results indicated that LIN28B regulated stem-like pluripotency and EMT phenotypes to promote t-NEPC development.
LIN28B promotes t-NEPC tumorigenesis and tumor growth. The gain of a stem-like phenotype has been demonstrated to associate with PCa tumorigenesis and progression $(38,39)$. To study the impact of LIN28B on xenograft growth, we inoculated DuNE(gCTL) and DuNE(gLIN28B) cells subcutaneously into immunocompromised mice ( $n=16 /$ group). There were 6 DuNE(gLIN28B) cell inoculations that never generated a xenograft during the 3 months of the animal study. The remaining 10 DuNE(gLIN28B) cell inoculations gave rise to tumors, but 

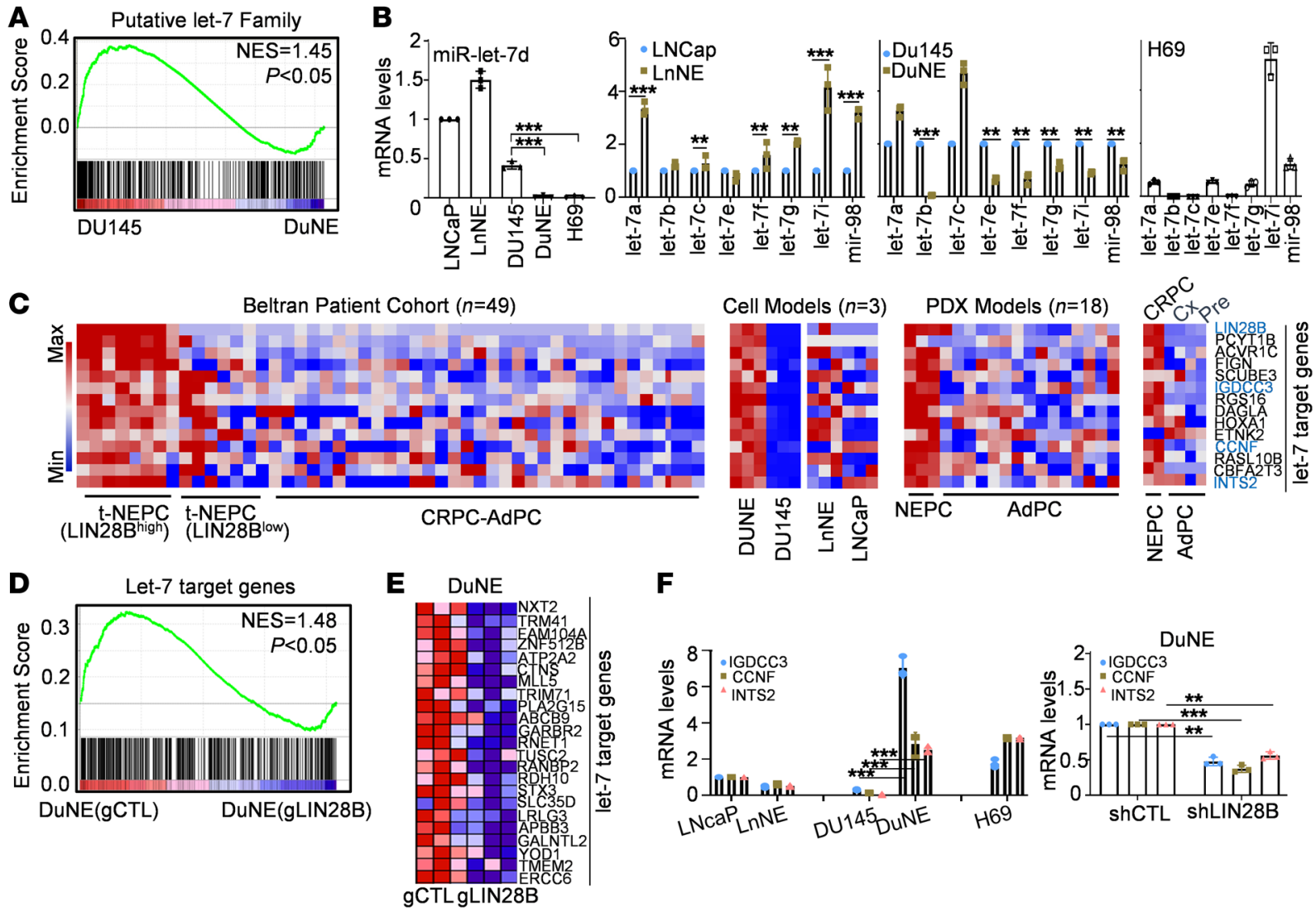

$\mathbf{F}$
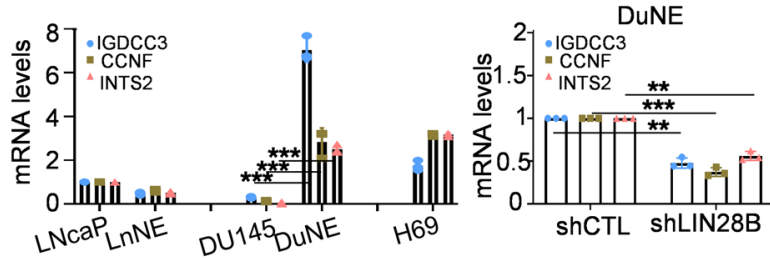

Figure 5. Let-7 is a negative downstream effector of LIN28B. (A) GSEA revealed the enrichment of the "putative let-7 family" signature in DU145 cells in comparison with DuNE cells. (B) Real-time PCR measured several let-7 family members in LNCaP, LnNE, DU145, DuNE, and NCI-H69 cells. (C) The expression of putative let-7 target genes identified from the leading-edge gene list from GSEA (A) was stratified from RNA-Seq results from the Beltran patient cohort, DuNE, and LnNE cell models and LTL331 PDX models. (D) GSEA showed the enrichment of the "let-7 target gene" signature in DuNE(gLIN28B) cells in comparison with DuNE(gCTL) cells. (E) The expression of putative let-7 target genes identified from the leading-edge gene list from GSEA (D) was plotted. (F) The mRNA levels of ICDCC3, CCNF, and INTS2 were measured in LNCaP, LnNE, DU145, DuNE, and NCI-H69 cells by real-time PCR. The Student's $t$ test was used to compare results between 2 groups with ${ }^{* *} P<0.01,{ }^{* *} P<0.001$.

the tumor take rates were significantly longer: the mean uptake time was 29 days compared with 20 days in the DuNE(gCTL) tumor group (Figure $4 \mathrm{~F}$ ). Even after the 10 tumors formed from the DuNE(gLIN28B) cells, their growth rate was much slower, having an average doubling time of 7.55 days (Figure $4 \mathrm{G}$ ), compared with DuNE(gCTL) tumors having an average doubling time of 5.13 days. Immunoblotting and IHC assays confirmed that DuNE(gLIN28B) tumors expressed residual LIN28B protein and reduced levels of $\mathrm{N}$-cadherin, CD44, SYP, and SOX2 (Figure $4, \mathrm{H}$ and I). Based on these results, we reasoned that LIN28B is important for t-NEPC initiation and progression. The residual LIN28B expression in the DuNE(gLIN28B) cells may be at a threshold that accounts for either no tumor development or a slower tumor progression rate.

LIN28B regulates let-7 signaling in PCa cells. The oncogenic role of the LIN28B/let-7 pathway has been established in various cancers, where the upregulation of LIN28B and the resultant downregulation of let-7 were correlated with poor prognosis and advanced malignancies (40). LIN28B-mediated inhibition of let-7 regulates tumor-initiating and self-renewal properties of CSCs (41). Although we recognize that there are other signal pathways (e.g., NF-кB,
REST, and RAS) through which LIN28B can act independently of let-7 (41), our results did not support these mechanisms (data not shown); rather, LIN28B-mediated suppression of let-7 was strongly implicated in promoting t-NEPC development (Figure 5). First, GSEA showed that DuNE cells were negatively associated with the "putative let-7 family" signature when compared with DU145 cells (Figure 5A). DuNE cells expressed significantly lower levels of several let-7 family members, with let-7d being the most strongly suppressed in the DuNE cells (Figure 5B). Second, RNA-Seq data from the Beltran patient cohort showed that a panel of let-7-suppressed genes from the leading-edge gene list predicted by GSEA (Figure $5 \mathrm{~A}$ ) were upregulated in the $\mathrm{t}-\mathrm{NEPC}\left(\mathrm{LIN} 28 \mathrm{~B}^{\mathrm{hi}}\right)$ in comparison with $\mathrm{t}-\mathrm{NEPC}\left(\mathrm{LIN} 28 \mathrm{~B}^{\mathrm{lo}}\right)$ and AdPC patient tumors (Figure $5 \mathrm{C}$ ). These expression patterns were replicable in the DuNE models and several t-NEPC PDXs, including the LTL331R model that progressed from the LTL331 tumors (Figure 5, C-E). Let-7-suppressed genes, including IGDCC3, CCNF, and INTS2, were highly expressed only in DuNE and NCI-H69 cells, both of which possess NE phenotypes (Figure 5F). Let-7 sponge (spg-let-7), which acts as a let-7 inhibitor, increased IGDCC3, CCNF, and INTS2 mRNA levels in DU145 cells, while a let-7d mimic inhibited the expression of these genes 
A

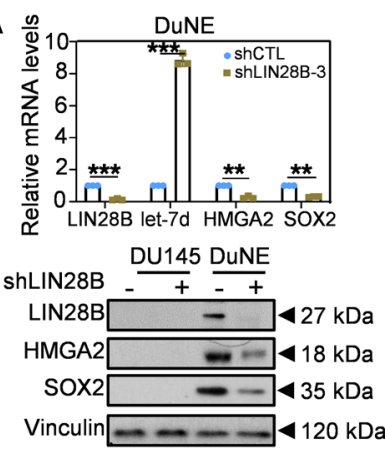

B

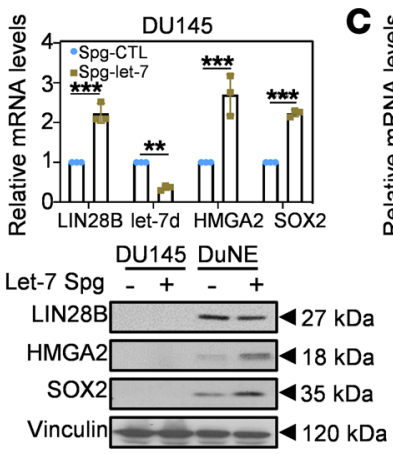

C $\frac{\infty}{0} 1.3 \times 104$ DuNE

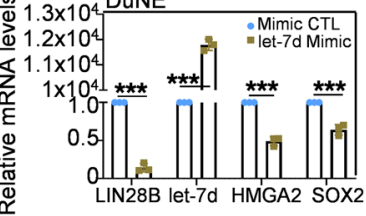

DU145 DUNE

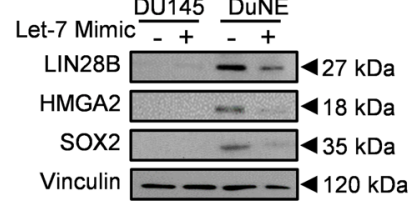

D

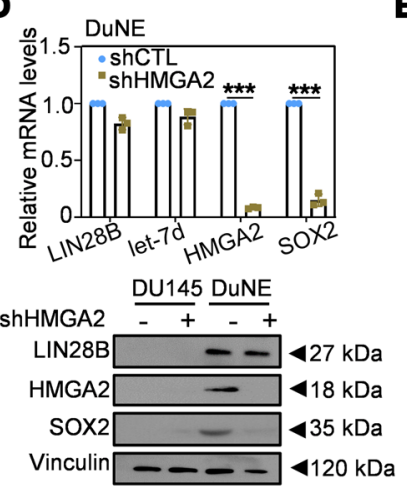

E
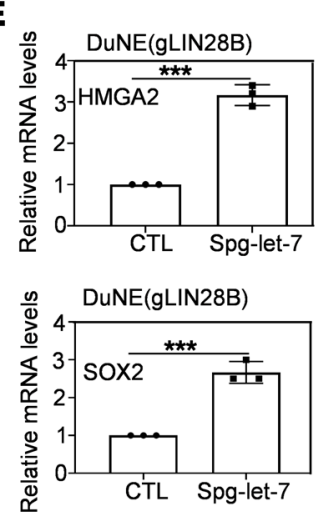

$\mathbf{F}$
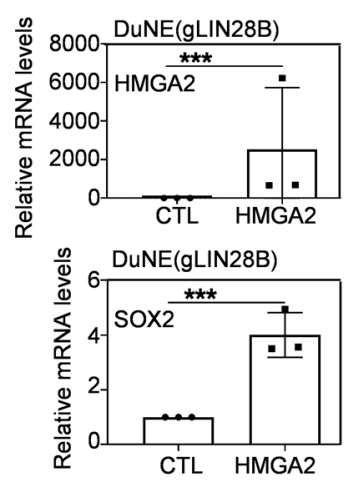

G

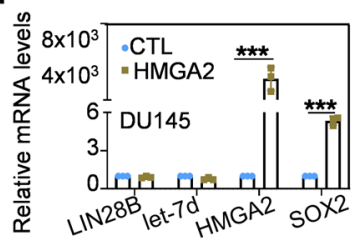

H

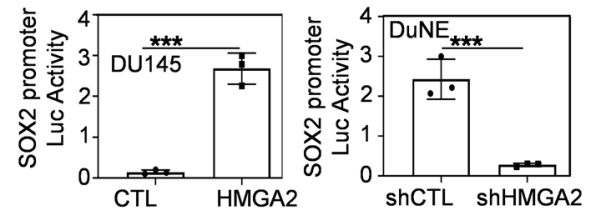

Figure 6. HMGA2 mediates LIN28B/let-7 signaling to induce SOX2 expression. DU145 and DuNE cells were transfected with control or shLIN28B (A); control or let-7 sponge vector (Addgene plasmid 29766) (B); control or let-7d mimic (Dharmacon) (c); control or ShHMGA2 vector (D). The mRNA and protein levels of LIN28B, HMCA2, and SOX2 were measured by real-time PCR and immunoblotting assays. DnNE(gLIN28B) cells were transfected with the let-7 sponge vector (E) or HMCA2 expression vector (F). HMCA2 and SOX2 mRNAs were measured by qPCR. (C) DU145 cells were transfected with control or HMGA2 expression vector. LIN28B, let-7, HMGA2, and SOX2 mRNAs were measured by qPCR. (H) DU145 and DuNE cell lines were transfected with HMCA2 or shHMGA2 vector, respectively. The luciferase reporter assays measured the SOX2 promoter activity. Three independent biological replicates were performed for each experiment. Only 1 set of representative immunoblots is shown. All results are presented as mean $\pm S D$. Statistical analyses were performed by 1-way ANOVA or unpaired Student's $t$ test with ${ }^{* *} P<0.01,{ }^{* * *} P<0.001$, respectively. (I) Kaplan-Meier plots showed progression-free survival of CRPC patients from the Grasso 2012 cohort after the first hormonal therapy in association with SOX2, HMGA2, and LIN28B expression. The Student's $t$ test was used to compare results between 2 groups.
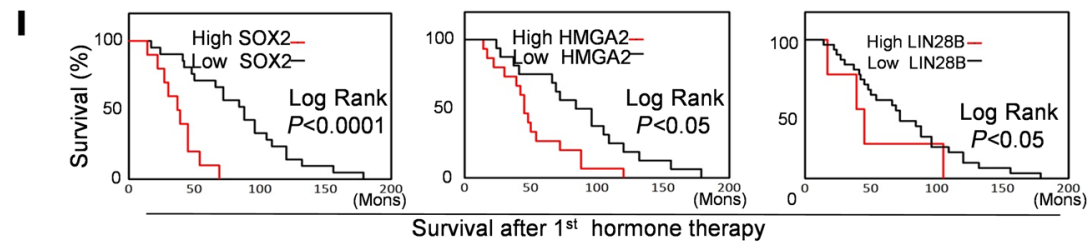

in DuNE cells (Supplemental Figure 8). Collectively, these results demonstrated that LIN28B regulated a stem-like gene network via let-7 miRNA for t-NEPC development.

HMGA 2 mediates LIN28B/let-7 signaling to induce SOX 2 expression. The association of LIN28B and SOX2 co-upregulation with t-NEPC progression suggests that LIN28B/let-7 signaling may stimulate SOX2 expression in contributing to t-NEPC development. The mechanism by which the LIN28B/let-7 axis induces SOX2 expression warrants further investigation. Although several signaling pathways (e.g., HMGA2, MYC, RAS, and EZH2) were reported to be the downstream targets of LIN28/let-7 (40, 42, 43), we found that LIN28B depletion in DuNE cells resulted in the suppression of HMGA2 and SOX2 at both MRNA and protein levels (Figure 6A). Consistently, spg-let-7 increased HMGA2 and SOX2 mRNA levels in DU145 cells and substantially enhanced HMGA2 and SOX2 protein levels in DuNE cells (Figure 6B). In contrast, let-7d mimic decreased the expression of HMGA2 and SOX2 expression in DuNE cells (Figure 6C), indicating that LIN28B/ let-7 signaling regulated HMGA2 expression in PCa cells. This finding was consistent with previous reports in hematopoietic stem cells and HeLa cells $(43,44)$. HMGA2 is an oncofetal protein that is highly expressed during embryonic development but is expressed at either very low levels or not at all in normal adult tissues. HMGA2 has oncogenic properties involved in tumor cell development, differentiation, stem cell self-renewal of CSCs, and EMT (45). Since HMGA2 is a transcription factor recruited to the SOX2 promoter to regulate SOX2 transcription (23), we sought to define the regulatory interactions between HMGA2 and SOX2 in PCa cells. HMGA2 knockdown in DuNE cells suppressed SOX2 (Figure 6D). Rescue experiments showed that spg-let-7 in DuNE(gLIN28B) cells upregulated HMGA2 and SOX2 expression (Figure 6E) and HMGA2 in DuNE(gLIN28B) cells enhanced SOX2 expression (Figure 6F). Consistently, overexpression of HMGA2 in DU145 cells enhanced SOX2 expression (Figure 6G). Further- 
more, luciferase reporter assays confirmed that HMGA2 overexpression in DU145 cells stimulated, whereas HMGA2 silencing in DuNE cells reduced, SOX2 promoter activity (Figure $6 \mathrm{H}$ ). These results demonstrated that $\mathrm{HMGA} 2$ is a downstream mediator of LIN28B/let-7 signaling to regulate SOX2 expression in PCa cells. Furthermore, we showed that high LIN28B, HMGA2, and SOX2 expression were all associated with progression-free survival of patients who received androgen deprivation therapy (Figure 6I), emphasizing that the LIN28B/HMGA2/SOX axis may be a biomarker of worse prognosis.

\section{Discussion}

Progression of AdPC to t-NEPC is an adaptive response of cancer cells acquiring lineage plasticity in order to escape anticancer treatments. This study defines a molecular mechanism by which LIN28B signaling confers PCa cell lineage plasticity to promote $\mathrm{t}-\mathrm{NEPC}$ progression through the regulation of a stem-like gene network. Based on these findings, inhibitors that block LIN28B signaling may help delay the emergence and/or progression of t-NEPC.

LIN28B was found to be highly expressed in approximately $40 \%-50 \%$ of CRPC-NEPC patient samples, which was based on the RNA-Seq results from the Beltran 2016 cohort (Figure 1) and the IHC results from the VPC cohort (Figure 2). However, an interesting perspective is that both RNA-Seq and IHC results represented a snapshot of the whole process of CRPC-AdPC progression to NEPC-CRPC. LIN28B expression may possibly be upregulated to initiate a CSC phenotype programming, and subsequently reduced after tumor cells complete transformation to $\mathrm{NE}$ lineage, in which case the number of CRPC-NEPC tumors driven by LIN28B could be underestimated.

Our results support the idea that LIN28B promotes a CSC-like phenotype of PCa cells and facilitates an NE lineage switch, rather than that LIN28B-positive PCa cells are canonical CSCs (46). Canonical CSCs commonly exist at low subpopulations within tumors and have limited proliferation activity distinct from nonCSCs that make up the bulk of the tumor. CSCs can undergo symmetrical divisions that give rise to CSCs, or asymmetrical divisions that give rise to CSCs and progenitor cells and contribute to tumor heterogeneity in a hierarchical organization. Our results showed that LIN28B-positive cells are highly populated in t-NEPC (Figure 2 ). When present, these cells are highly proliferative and express strong NE lineage markers (Figure 3). Previous studies have also reported that cancer cells, regardless of the presence of CSCs, are plastic and can undergo a phenotypic transition (47). This was exemplified by FACS-sorted breast cancer cells with a stem-like, basal, or luminal phenotype, where each subpopulation was capable of gaining phenotypes of the other 2 subpopulations (47). Consistent with this notion, when engineered by 5 gene factors, TP53, RB1, myrAKT1, c-Myc, and BCL2, normal prostatic basal epithelium gave rise to NEPC xenografts (48). Moreover, prostate luminal cells can also gain stem-like, mesenchymal, and NE phenotypes by AR inhibition and progress to t-NEPC $(7,8)$. Collectively, our study identified LIN28B as an oncogenic factor that regulated $\mathrm{PCa}$ cell plasticity to promote t-NEPC progression.

Gaining stem-like phenotypes is likely a common mechanism that is associated with CRPC progression. This phenotype could be manifested by either increased expression of stemness genes (e.g., CD44, CD133, and BMI1), reduced expression of luminal epithelial biomarkers (e.g., AR and PSA), or an altered expression of EMT-related genes (e.g., E-CAD and SNAI2), all of which are associated with castration resistance and metastasis (49-52). It is worth noting that the LIN28A isoform was reported to be overexpressed in CRPC-AdPC, where it stimulates AdPC cell proliferation, anchorage-independent colony formation, and tumorigenesis (53). More importantly, LIN28A enhances the expression of luminal epithelial biomarkers, including AR, PSA, and NKX3.1, as well as AR-v7 (54). Because LIN28A and LIN28B genes are usually not coexpressed in the same cell lineage (25), these results indicate that LIN28A may promote AdPC CRPC progression that maintains the epithelium lineage, while gain of function of LIN28B promotes CRPC progression toward t-NEPC.

Our results from LIN28B gene CRISPR studies and results from other investigators $(53,54)$ have shown that targeting LIN28 isoforms and their stem-like gene networks may be beneficial to CRPC patients with either t-NEPC or AdPC phenotypes. Furthermore, LIN28 signaling contributing to therapy resistance has been demonstrated in many types of tumors (55-59). Since both LIN28B and LIN28A share homology in structure, efforts have been made to screen small-molecule inhibitors blocking LIN28 from recognizing let-7 miRNAs (60-62). Testing these compounds in CRPC cell and xenograft models and/or designing novel LIN28 inhibitors by applying in silico guided drug design would accelerate the translation of LIN28 into targeted therapy.

In summary, our study reveals a molecular mechanism by which t-NEPC can develop through CSC-like signaling mediated by LIN28B and proposes targeting this signaling as an anticancer therapy.

\section{Methods}

RNA-Seq data from patients, PDXs, GEMMs, and cell models. Clinical cohorts used in this study include the following: RNA-Seq data for the Beltran 2016 cohort (CRPC-Ad, $n=34$; t-NEPC, $n=15$ ) were from Weill Medical College of Cornell University (30). RNA-Seq data for the DuNE and LnNE cell models were previously reported by our lab $(31,32)$. LTL PDX data were accessed in NCBI's Gene Expression Omnibus (GEO) database (GSE59986) (63). Sequencing data for the GEMMs, including Ku et al. (WT, SKO, DKO, TKO), were accessed in GEO GSE90891 (8). RNA-Seq data for the SU2C 2015 cohort (CRPC-Ad, $n=113$; CRPC$\mathrm{NE}, n=5$ ) were accessed through cBioPortal. Data from the Zou et al. (NPp53) cohort were accessed in GEO GSE92721 (15).

Cell lines and cell culture. Prostate cancer cell lines (LNCaP, C4-2, RWPE-1, 22RV1, PC-3, VCaP, Du145, NCI-H660, and LASCPC) and small cell lung cancer cell lines (NCI-H82 and NCI-H69) were purchased from American Type Culture Collection. LNCaP95 cells were provided by Alan Meeker (Johns Hopkins University, Baltimore, Maryland, USA). MR42D and MR42F cells were provided by Amina Zoubeidi from the VPC. LnNE and DuNE cells were reported from our previous studies (31, $32)$. All cell culture conditions have been previously reported $(12,14)$. All lines used in this study tested negative for mycoplasma contamination and were authenticated by short tandem repeat assays.

CRPC TMA and IHC analyses. The CRPC TMA containing 100 tissue cores from 50 patients who had undergone hormonal therapy, chemotherapy, or radiotherapy was obtained from the tissue bank at the VPC. Histopathology of the primary tumors $(n=16)$, CRPC $(n=54)$, and t-NEPC tumors $(n=30)$ has been previously 
reported and characterized (32). IHC was performed by using a Ventana Discovery XT autostainer as previously described $(12,36$, 37). Briefly, slides in citrate buffer $(\mathrm{pH}=6)$ were heated in a steamer for 30 minutes. After cooling and washing, the slides were incubated in $3 \% \mathrm{H}_{2} \mathrm{O}_{2}$ for 10 minutes, blocked with 3\% BSA for 30 minutes, and then incubated with specific antibodies (LIN28B, Abcam ab71415, 1:25 dilution; SOX2, Novus NB110-37235, 1:25 dilution). The slides were washed extensively with PBS and examined with UltraMap DAB anti-Rb Detection Kit (Ventana). The SL801 autoloader and the Leica SCN400 scanning system (Leica Microsystems) were used to digitize the slides at an original magnification of $\times 40$ (scale bar: $100 \mu \mathrm{m}$ ). The images were subsequently stored in the SlidePath Digital Imaging Hub (Leica Microsystems) at the VPC. Pathological evaluation of these tumors was performed independently by 3 pathologists. All antibodies used are listed in Supplemental Tables 1-3.

Standard molecular techniques. Standard techniques, including plasmid DNA or siRNA transfection, real-time PCR, immunoblotting, luciferase reporter assays, FACS, colony formation, MTS assays, and Ion AmpliSeq Transcriptome Sequencing, were performed as previously reported $(12,14,31,32)$. Information about oligo primers and antibodies used is listed in Supplemental Tables 1-3. Antibody and primer information, including sources, catalog numbers, and working conditions, is described in Supplemental Tables 1-3.

GeneArt CRISPR technology. DuNE cells with LIN28B knockout were constructed by using the GeneArt Precision gRNA Synthesis Kit (Invitrogen, Thermo Fisher Scientific, A29377) and GeneArt Platinum Cas9 Nuclease (Invitrogen, Thermo Fisher Scientific, B25640) according to the manufacturer's protocol. The sequences for gRNA synthesis were designed using the GeneArt CRISPR Search and Design tool (https://www.thermofisher.com/us/en/home/life-science/genome-editing/geneart-crispr/geneart-crispr-search-and-design-tool.html). Cells underwent selection for single-cell colonies in 96-well plates. Mutations that disrupted LIN28B expression were confirmed by Sanger sequencing and immunoblotting assays.

Xenograft studies in nude mice. To generate DuNE xenografts, $1 \times$ $10^{6}$ cells from the DuNE(gCTL) and DuNE(gLIN28B) cell lines were implanted subcutaneously in the bilateral flanks of 6- to 8-week-old male nude mice $(n u / n u)$. Tumor volume $(\mathrm{V}=$ length $\times$ width $\times$ height $\times$ 0.5236) was measured 3 times a week.

Statistics. Statistical analysis was performed using the GraphPad Prism 6.0 software (GraphPad Software). Experimental data were normalized to internal controls from at least 3 independent biological replicates with all data represented as means \pm SD. When comparing data from 2 treatment groups (e.g., comparisons between \pm LIN28B CRISPR), a Student's 2-tailed $t$ test was used to determine significance, a $P$ value less than 0.05. When comparing more than 2 groups, ANOVA with Tukey's test for pairwise comparisons was used among different experimental groups. Spearman's correlation coefficient and the corresponding $P$ value were used to measure the extent of correlation between LIN28B and SOX2 histology scores. The level of significance was set at ${ }^{*} P<0.05,{ }^{* *} P<0.01$, and ${ }^{* *} P<0.001$.

Study approval. The VPC cohort research protocol was approved by the Clinical Research Ethics Board of University of British Columbia (UBC) (H09-01628). All patients signed a formal consent form approved by the ethics board. All animal procedures were performed under the guidelines of the Canadian Council on Animal Care and were approved by the UBC Animal Care Committee (A19-0256).

\section{Author contributions}

JL, YG, and XD designed the research. JL, YG, TF, YL, NX, CH, and ARL performed the research. JL, YG, XC, BH, LF, and JH analyzed the data. LN and MEG shared research materials. XD and MEG wrote the manuscript. YG developed the hypothesis and initiated this project by collecting data at the early stage of this project; JL finalized the project by collecting the majority of the experimental data. The order of these co-first authors is based on the length of time spent on the project.

\section{Acknowledgments}

The authors would like to thank Sonia Kung from the Pathology Core and Mary Bowden from the animal facility at the Vancouver Prostate Centre for their technical support. We thank Nathan Lack, Ralph Buttyan, and Amina Zoubeidi for their insightful discussion of this project. This project was supported by operating grants from the Canadian Institutes of Health Research (MOP137007, PJT-156150 to XD), Terry Fox Research Institute New Frontiers program grant (1062 to XD and MEG), U.S. Department of Defense (W81XWH2010581 to XD and MEG), National Natural Science Foundation of China (81902606 to YG), Nature Science Foundation of Hunan Province (2020JJ5891 to YG), and China Scholarship Council fellowships to YG, YL, and TF.

Address correspondence to: Xuesen Dong, 2660 Oak Street, Vancouver, British Columbia, Canada V6H 3Z6. Phone: 604.875.4111; Email: xdong@prostatecentre.com.
1. Hirano D, Okada Y, Minei S, Takimoto Y, Nemoto N. Neuroendocrine differentiation in hormone refractory prostate cancer following androgen deprivation therapy. Eur Urol. 2004;45(5):586-592.

2. Sasaki T, et al. Changes in chromogranin a serum levels during endocrine therapy in metastatic prostate cancer patients. Eur Urol. 2005;48(2):224-229.

3. Bluemn EG, et al. Androgen receptor pathway-independent prostate cancer is sustained through FGF signaling. Cancer Cell. 2017;32(4):474-489.e6.

4. Aggarwal R, et al. Clinical and genomic characterization of treatment-emergent small-cell neuroendocrine prostate cancer: a multi-in- stitutional prospective study. JClin Oncol. 2018;36(24):2492-2503.

5. Beltran $\mathrm{H}$, et al. The role of lineage plasticity in prostate cancer therapy resistance. Clin Cancer Res. 2019;25(23):6916-6924.

6. Wang HT, Yao YH, Li BG, Tang Y, Chang JW, Zhang J. Neuroendocrine prostate cancer (NEPC) progressing from conventional prostatic adenocarcinoma: factors associated with time to development of NEPC and survival from NEPC diagnosis-a systematic review and pooled analysis. J Clin Oncol. 2014;32(30):3383-3390.

7. $\mathrm{Mu} \mathrm{P}$, et al. SOX2 promotes lineage plasticity and antiandrogen resistance in TP53- and RB1-deficient prostate cancer. Science.
2017;355(6320):84-88.

8. Ku SY, et al. Rb1 and Trp53 cooperate to suppress prostate cancer lineage plasticity, metastasis, and antiandrogen resistance. Science. 2017;355(6320):78-83.

9. Zou M, et al. Transdifferentiation as a mechanism of treatment resistance in a mouse model of castration-resistant prostate cancer. Cancer Discov. 2017;7(7):736-749.

10. Beltran H, et al. Molecular characterization of neuroendocrine prostate cancer and identification of new drug targets. Cancer Discov. 2011;1(6):487-495.

11. Berger A, et al. N-Myc-mediated epigenetic reprogramming drives lineage plasticity 
in advanced prostate cancer. J Clin Invest. 2019;129(9):3924-3940.

12. Li Y, et al. RNA splicing of the BHC80 gene contributes to neuroendocrine prostate cancer progression. Eur Urol. 2019;76(2):157-166.

13. Dardenne E, et al. N-Myc induces an EZH2-mediated transcriptional program driving neuroendocrine prostate cancer. Cancer Cell. 2016;30(4):563-577.

14. Li Y, et al. SRRM4 drives neuroendocrine transdifferentiation of prostate adenocarcinoma under androgen receptor pathway inhibition. Eur Urol. 2017;71(1):68-78.

15. Bishop JL, et al. The master neural transcription factor BRN2 is an androgen receptor-suppressed driver of neuroendocrine differentiation in prostate cancer. Cancer Discov. 2017;7(1):54-71.

16. Aggarwal RR, et al. Whole-genome and transcriptional analysis of treatment-emergent small-cell neuroendocrine prostate cancer demonstrates intraclass heterogeneity. Mol Cancer Res. 2019;17(6):1235-1240.

17. $\mathrm{Yu}$ J, et al. Induced pluripotent stem cell lines derived from human somatic cells. Science. 2007;318(5858):1917-1920.

18. Cai WY, et al. The Wnt- $\beta$-catenin pathway represses let-7 microRNA expression through transactivation of Lin28 to augment breast cancer stem cell expansion. J Cell Sci. 2013;126(pt 13):2877-2889.

19. Kong D, et al. Loss of let-7 up-regulates EZH2 in prostate cancer consistent with the acquisition of cancer stem cell signatures that are attenuated by BR-DIM. PLoS One. 2012;7(3):e33729.

20. Meirelles K, et al. Human ovarian cancer stem/ progenitor cells are stimulated by doxorubicin but inhibited by Mullerian inhibiting substance. Proc Natl Acad Sci U S A. 2012;109(7):2358-2363.

21. Yang $X$, et al. Double-negative feedback loop between reprogramming factor LIN28 and microRNA let-7 regulates aldehyde dehydrogenase 1-positive cancer stem cells. Cancer Res. 2010;70(22):9463-9472.

22. Zhang WC, et al. Glycine decarboxylase activity drives non-small cell lung cancer tumor-initiating cells and tumorigenesis. Cell. 2012;148(1-2):259-272.

23. Chien CS, et al. Lin28B/Let-7 regulates expression of Oct 4 and Sox 2 and reprograms oral squamous cell carcinoma cells to a stem-like state. Cancer Res. 2015;75(12):2553-2565.

24. Cimadamore F, Amador-Arjona A, Chen C, Huang CT, Terskikh AV. SOX2-LIN28/let-7 pathway regulates proliferation and neurogenesis in neural precursors. Proc Natl Acad Sci U S A. 2013;110(32):E3017-E3026.

25. Piskounova E, et al. Lin28A and Lin28B inhibit let-7 microRNA biogenesis by distinct mechanisms. Cell. 2011;147(5):1066-1079.

26. Heo I, et al. TUT4 in concert with Lin28 suppresses microRNA biogenesis through pre-microRNA uridylation. Cell. 2009;138(4):696-708.

27. Qiu C, Ma Y, Wang J, Peng S, Huang Y. Lin28mediated post-transcriptional regulation of
Oct4 expression in human embryonic stem cells. Nucleic Acids Res. 2010;38(4):1240-1248.

28. Jin J, et al. Evidence that Lin28 stimulates translation by recruiting RNA helicase A to polysomes. Nucleic Acids Res. 2011;39(9):3724-3734.

29. Närvä E, et al. RNA-binding protein L1TD1 interacts with LIN28 via RNA and is required for human embryonic stem cell self-renewal and cancer cell proliferation. Stem Cells. 2012;30(3):452-460.

30. Beltran H, et al. Divergent clonal evolution of castration-resistant neuroendocrine prostate cancer. Nat Med. 2016;22(3):298-305.

31. Li Y, et al. Establishment of a neuroendocrine prostate cancer model driven by the RNA splicing factor SRRM4. Oncotarget. 2017;8(40):66878-66888.

32. Lee AR, Gan Y, Tang Y, Dong X. A novel mechanism of SRRM4 in promoting neuroendocrine prostate cancer development via a pluripotency gene network. EBioMedicine. 2018;35:167-177.

33. Lin D, et al. High fidelity patient-derived xenografts for accelerating prostate cancer discovery and drug development. Cancer Res. 2014;74(4):1272-1283.

34. Barretina J, et al. The Cancer Cell Line Encyclopedia enables predictive modelling of anticancer drug sensitivity. Nature. 2012;483(7391):603-607.

35. Chang TC, et al. Lin-28B transactivation is necessary for Myc-mediated let-7 repression and proliferation. Proc Natl Acad Sci U S A. 2009;106(9):3384-3389.

36. Li Y, et al. SRRM4 gene expression correlates with neuroendocrine prostate cancer. Prostate. 2019;79(1):96-104.

37. Guo $\mathrm{H}$, et al. ONECUT2 is a driver of neuroendocrine prostate cancer. Nat Commun. 2019;10(1):278.

38. Tang DG. Understanding cancer stem cell heterogeneity and plasticity. Cell Res. 2012;22(3):457-472.

39. Zhang D, Tang DG, Rycaj K. Cancer stem cells: regulation programs, immunological properties and immunotherapy. Semin Cancer Biol. 2018;52(Pt 2):94-106.

40. Viswanathan SR, et al. Lin28 promotes transformation and is associated with advanced human malignancies. Nat Genet. 2009;41(7):843-848.

41. Balzeau J, Menezes MR, Cao S, Hagan JP. The LIN28/let-7 pathway in cancer. Front Genet. 2017;8:31.

42. Oshima M, et al. Ezh2 regulates the Lin28/ let-7 pathway to restrict activation of fetal gene signature in adult hematopoietic stem cells. Exp Hematol. 2016;44(4):282-96.e3.

43. Copley MR, et al. The Lin28b-let-7-Hmga2 axis determines the higher self-renewal potential of fetal haematopoietic stem cells. Nat Cell Biol. 2013;15(8):916-925

44. Lee YS, Dutta A. The tumor suppressor microRNA let-7 represses the HMGA2 oncogene. Genes Dev. 2007;21(9):1025-1030.

45. Young AR, Narita M. Oncogenic HMGA2: short or small? Genes Dev. 2007;21(9):1005-1009.

46. Batlle E, Clevers H. Cancer stem cells revisited.
Nat Med. 2017;23(10):1124-1134.

47. Gupta PB, et al. Stochastic state transitions give rise to phenotypic equilibrium in populations of cancer cells. Cell. 2011;146(4):633-644.

48. Park JW, et al. Reprogramming normal human epithelial tissues to a common, lethal neuroendocrine cancer lineage. Science. 2018;362(6410):91-95.

49. Chen X, et al. Defining a population of stem-like human prostate cancer cells that can generate and propagate castration-resistant prostate cancer. Clin Cancer Res. 2016;22(17):4505-4516.

50. Stylianou N, et al. A molecular portrait of epithelial-mesenchymal plasticity in prostate cancer associated with clinical outcome. Oncogene. 2019;38(7):913-934.

51. Li Q, et al. Linking prostate cancer cell AR heterogeneity to distinct castration and enzalutamide responses. Nat Commun. 2018;9(1):3600.

52. Yoo YA, Vatapalli R, Lysy B, Mok H, Desouki MM, Abdulkadir SA. The role of castration-resistant Bmi1+Sox 2+ cells in driving recurrence in prostate cancer. J Natl Cancer Inst. 2019;111(3):311-321.

53. Tummala R, et al. Lin 28 promotes growth of prostate cancer cells and activates the androgen receptor. Am J Pathol. 2013;183(1):288-295.

54. Tummala R, Nadiminty N, Lou W, Evans CP, Gao AC. Lin28 induces resistance to anti-androgens via promotion of AR splice variant generation. Prostate. 2016;76(5):445-455.

55. Lv K, et al. Lin28 mediates paclitaxel resistance by modulating $\mathrm{p} 21, \mathrm{Rb}$ and Let-7a miRNA in breast cancer cells. PLoS One. 2012;7(7):e40008.

56. Leprivier $\mathrm{G}$, et al. The eEF2 kinase confers resistance to nutrient deprivation by blocking translation elongation. Cell. 2013;153(5):1064-1079.

57. Tian N, Han Z, Li Z, Zhou M, Fan C. Lin28/let-7/ Bcl-xL pathway: the underlying mechanism of drug resistance in Hep3B cells. Oncol Rep. 2014;32(3):1050-1056.

58. Oh JS, Kim JJ, Byun JY, Kim IA. Lin28-let7 modulates radiosensitivity of human cancer cells with activation of K-Ras. Int J Radiat Oncol Biol Phys. 2010;76(1):5-8

59. Ma JX, et al. PSP94, an upstream signaling mediator of prostasin found highly elevated in ovarian cancer. Cell Death Dis. 2014;5:e1407.

60. Wang L, et al. Small-molecule inhibitors disrupt let-7 oligouridylation and release the selective blockade of let-7 processing by LIN28. Cell Rep. 2018;23(10):3091-3101.

61. Lim D, Byun WG, Park SB. Restoring let-7 microRNA biogenesis using a small-molecule inhibitor of the protein-RNA interaction. ACS Med Chem Lett. 2018;9(12):1181-1185.

62. Roos M, et al. A small-molecule inhibitor of Lin28. ACS Chem Biol. 2016;11(10):2773-2781.

63. Akamatsu S, et al. The placental gene PEG10 promotes progression of neuroendocrine prostate cancer. Cell Rep. 2015;12(6):922-936.

64. Ci X, et al. Heterochromatin protein $1 \alpha$ mediates development and aggressiveness of neuroendocrine prostate cancer. Cancer Res. 2018;78(10):2691-2704. 